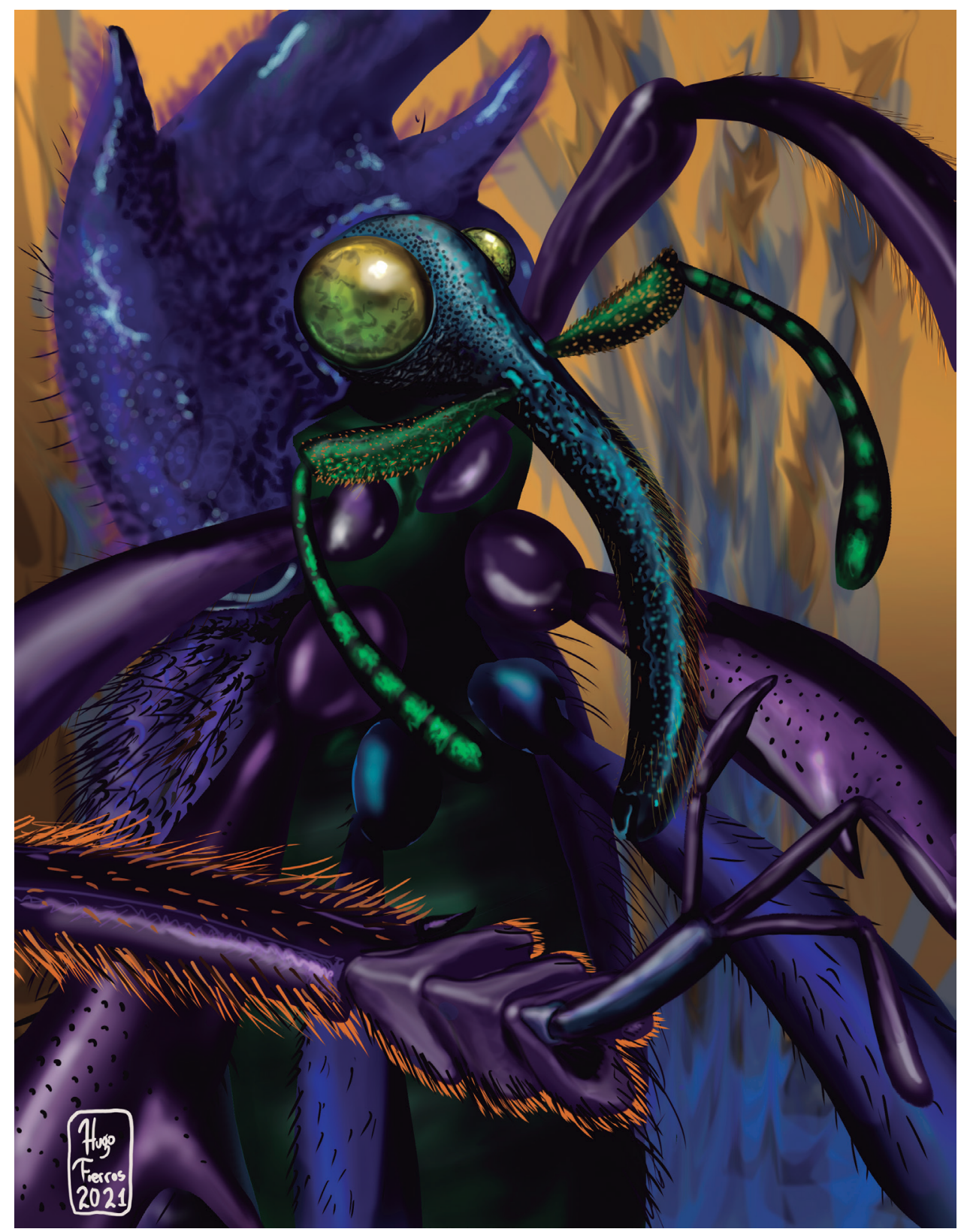

Dugesiana, Año 28, No. 2, (julio-diciembre, segundo semestre de 2021), es una publicación semestral, editada por la Universidad de Guadalajara, a través del Centro de Estudios en Zoología, por el Centro Universitario de Ciencias Biológicas y Agropecuarias. Camino Ramón Padilla Sánchez \# 2100, Nextipac, Zapopan, Jalisco, Tel. 37771150 ext. 33218, http://148.202.248.171/dugesiana/index.php/DUG/index, glenusmx@gmail.com. Editor responsable: José Luis Navarrete-Heredia. Reserva de Derechos al Uso Exclusivo 04-2009-062310115100-203, ISSN: 2007-9133, otorgados por el Instituto Nacional del Derecho de Autor. Responsable de la última actualización de este número: José Luis Navarrete-Heredia, Editor y Ana Laura González-Hernández, Asistente Editorial. Fecha de la última modificación 1 de julio de 2021, con un tiraje de un ejemplar.

Las opiniones expresadas por los autores no necesariamente reflejan la postura del editor de la publicación.

Queda estrictamente prohibida la reproducción total o parcial de los contenidos e imágenes de la publicación sin previa autorización de la Universidad de Guadalajara. 
Artículo

\title{
Los ejemplares tipo de Insecta depositados en la Colección Entomológica Conservada en Alco- hol del Instituto-Fundación Miguel Lillo, Tucumán, Argentina
}

\section{The type specimens of Insecta deposited in the Entomological Collection Conserved in Alcohol of the Instituto-Fundación Miguel Lillo, Tucumán, Argentina}

\author{
Silvia Patricia Córdoba \\ Instituto de Entomología, Área de Zoología, Fundación Miguel Lillo, Miguel Lillo 251 (4000), San Miguel de \\ Tucumán, Argentina., spcordoba@lillo.org.ar, ORCID: https://orcid.org/0000-0002-4269-3170
}

\section{RESUMEN}

La Colección Entomológica del Instituto- Fundación Miguel Lillo (I- FML) tiene cerca de 2.000.000 de ejemplares, entre los cuales hay 4.046 especímenes tipo que corresponden a 105 especies. El material depositado viene de los siguientes países: Argentina, Bolivia, Brasil, Chile, Colombia, Ecuador, Honduras, México, Perú, Uruguay y Venezuela. El objetivo del presente trabajo es aportar al conocimiento de los ejemplares de la serie tipo de los órdenes Diptera, Hymenoptera, Isopetra, Plecoptera, Trichoptera y Zygentoma, depositados en la colección entomológica conservada en alcohol del I- FML.

Palabras clave: Insectos, holotipo, alotipo, paratipo, catálogo.

\begin{abstract}
The Entomological Collection of Miguel Lillo Foundation- Institute (I- FML) has about 2.000.000 specimens, among whichthere are 4.046 type specimens corresponding to 105 species. The deposites material comes from the following countries: Argentina, Bolivia, Brasil, Chile, Colombia, Ecuador, Honduras, México, Perú, Uruguay and Venezuela. The objetive of this work is to contribute to the knowledge of the specimens of the type series of the orders Diptera, Hymenoptera, Isoptera, Plecoptera, Trichoptera and Zygentoma, deposited in the entomological collection preserved in alcohol of I-FML.
\end{abstract}

Key words: Insects, holotype, allotype, paratype, catalogue.

Los insectos comprenden el grupo de animales más diverso que habita la Tierra (Bustillo Pardey y Gil Palacios 2008). Más de la mitad (54\%) de todas las especies de organismos conocidos, y el $75 \%$ de todas las especies de animales son insectos (Zumbado y Azofeifa 2018). Son el grupo con mayor éxito evolutivo (Purvis y Hector 2000), principalmente por su abundancia, diversidad y el amplio espectro de hábitats y posiciones funcionales que ocupan (Llorente- Bouquets et al. 1996; Mattoni et al. 2000). Los insectos han sido un elemento importante no sólo por su función en los ecosistemas terrestres, sino también por su influencia en las sociedades humanas (Guzman- Mendoza, et al. 2016). Desde los albores de la humanidad estos organismos han sido parte de la alimentación, la salud, la cultura y el de los agroecosistemas no sólo como competidores, sino también como elementos pronosticadores y promotores de servicios ecosistémicos (Guzman- Mendoza et al. 2016).

La Colección Entomológica Conservada en Alcohol del Instituto- Fundación Miguel Lillo (I- FML) preserva aproximadamente 2.000.000 de ejemplares de insectos, con representantes de casi todos los órdenes, los cuales se mantienen en alcohol etílico (desde 70\% hasta 96\%) (Córdoba 2019), de los cuales 4.046 ejemplares son series tipo que corresponden a 105 especies, pertenecientes a ocho órdenes: Diptera, Hymenoptera, Isoptera, Ephemeroptera, Neuroptera, Plecoptera, Trichoptera y Zygentoma (=Thysanura). Estos ejemplares se encuentran conservados en tubos de Kahn o de vidrio, junto a los rótulos correspondientes a los de datos de procedencia, número de catálogo y determinación.

La mayoría de los catálogos de especímenes tipo publicados, se realizaron en base a los ejemplares conservados en la Colección Entomológica Conservada en Seco del IFML, excepto un primer aporte de los ejemplares tipo de Ephemeroptera depositados en la colección conservada en alcohol publicado por Córdoba (2019). Kenneth Hayward (1963), realizó una lista de los ejemplares tipo de Insecta depositados en la Colección Entomológica Conservada en Seco del I-FML, donde además incluyó los ejemplares tipo del orden Zygentoma conservados en alcohol.

El objetivo del presente trabajo es dar a conocer los ejemplares de la serie tipo de Diptera, Hymenoptera, Isoptera, Plecoptera, Trichoptera y Zygentoma depositados en la Colección Entomológica Conservada en Alcohol del IFML, como segundo aporte al conocimiento de dicha colección.

\section{MATERIAL Y MÉTODOS}

En el presente catálogo se incluyen los ejemplares de la 
serie tipo depositados en la Colección Entomológica Conservada en Alcohol de los órdenes Diptera, Hymenoptera, Isoptera, Plecoptera, Trichoptera, y Zygentoma, ubicada en el $3^{\circ}$ piso, Instituto de Entomología, Área de Zoología de la Fundación Miguel Lillo (FML). Se analizaron minuciosamente las etiquetas junto a los ejemplares de la serie tipo y las publicaciones originales. Para cada ejemplar se proporciona:

Nombre del taxón: los nombres de las especies se corresponden con los presentes en los rótulos, junto al material y con los de la publicación original. Se revisaron las actualizaciones taxonómicas y se reemplazaron los nombres de las especies en caso que hayan cambiado. Las familias están ordenadas alfabéticamente y dentro de estas, del mismo modo las especies.

Publicación original: se cita el autor, año de la publicación y página donde se describe la especie en cuestión.

Categoría taxonómica: se aclara la categoría, holotipo, alotipo o paratipo.

Sexo: se lo expresa cuando está indicado en el rótulo junto al ejemplar o en la publicación original.

Número de catálogo: corresponde al asignado cuando fue incorporado a la colección. Contiene las siglas: A: que corresponde a la colección conservada en alcohol, para diferenciarla del material conservado en la colección en seco; T: se les asigna a los ejemplares de la serie tipo y las tres siguientes letras se refieren a las tres primeras letras del nombre de orden al que pertenecen. Le sigue el número correlativo según el momento en que fue incorporado, comenzando con 001 en adelante.

Datos de recolección: país, provincia, localidad, coordenadas geográficas, altura, fecha de colecta, colector/es.

Número de ejemplares: número de especímenes por lote, en el caso de paratipos. Se aclara si se trata de ejemplares completos, exuvia, larva o pupa.

Observaciones: si fuera necesaria alguna aclaración, como cuando no hay concordancia entre la publicación original y el material depositado.

\section{RESULTADOS Diptera \\ Blephariceridae}

Limonicola hoguei Zwick, 2007: 109

HOLOTIPO ô (ATDIP002). Argentina, Prov. Jujuy, Par-

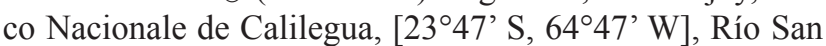
Lorenzo, 26/08/1998, Pharate $\hat{o}$ (parts on slide).

PARATIPO $\widehat{\jmath}$ (ATDIP003). Mismos datos que el Holotipo. 1 ejemplar.

PARATIPO $q$ (ATDIP004). Mismos datos que el Holotipo. Parts on micro slide. 1 ejemplar.

\section{Simulidae}

Gigantodax horcotiani Wygodzinski, 1949a: 326

PARATIPO (ATDIP001). Argentina, Tucumán, Tafí del Valle, 6000 m., 17/IX/1950, coll. Wygodzinski. 3 ejemplares.

\section{Hymenoptera \\ Mymaridae}

Acmopolynema commune Fidalgo, 1989: 15

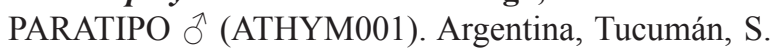
M. de Tucumán, VIII/1977, trampa Moericke, col. P. Fidalgo. 1 ejemplar.

PARATIPO $\widehat{\partial}$ (ATHYM002). Argentina, Tucumán, S. M. de Tucumán, 15- 30/IX/1981, trampa Moericke, col. P. Fidalgo. 1 ejemplar.

PARATIPO $\circlearrowright$ (ATHYM003). Argentina, Tucumán, S. M. de Tucumán. XII/1976, trampa Moericke, col. P. Fidalgo. 1 ejemplar.

PARATIPOS + (ATHYM004). Argentina, Tucumán, S. M. de Tucumán. 1-15/IX/1981, trampa Moericke, col. P. Fidalgo. 7 ejemplares.

PARATIPO + (ATHYM005). Argentina, Tucumán, S. M. de Tucumán. V/1976, trampa Moericke, col. P. Fidalgo. 1 ejemplar.

\section{Isoptera \\ Kalotermitidae}

Cryptotermes chacoensis Roisin, 2003: 320

PARATIPOS (ATISO001). Argentina, Parque Nacional Río Pilcomayo, S 25 02’ 45”, W 58 03’34”, 09/X/1999, col. Y. Roisin and M. Leponce. 3 ejemplares.

PARATIPOS (ATISO002). Argentina, Parque Nacional Río Pilcomayo, S 25 02’ 45”, W 58 03'34”, 12/X/1999, col. Y. Roisin and M. Leponce. 3 ejemplares.

Observaciones: los 6 paratipos fueron donados por el autor.

\section{Neuroptera \\ Coniopterygidae \\ Coniopteryx (Scotoconiopteryx) calileguana Sziráki, 2009: 177 \\ HOLOTIPO $\widehat{\text { }}$ (ATNEU030). Argentina, Prov. de Jujuy,} Dpto. Ledesma, Calilegua N P, S of Abra de Cañas, moss forest, S $23^{\circ} 41.3^{\prime}$, W $64^{\circ} 54.1^{\prime}, 2253$ m. a. s. 1., 30/10/2006, leg. Gy. Sziráki, E. Horvát, E. González Olazo.

PARATIPO $\sigma^{\wedge}$ (ATENU031). Argentina, Prov. de Jujuy, Dpto. Valle Grande, San Francisco, S $23^{\circ} 37.6^{\prime}$, W $64^{\circ}$ 56.2', 2084 m. a. s. 1., 29/10/2006, leg. Gy. Sziráki, E. Horvát, E. González Olazo. 1 ejemplar.

Incasemidalis pachamama, Sziráki, 2009:192

HOLOTIPO $\bigcirc$ (ATNEU022). Argentina, Catamarca, El Puesta (Caspinchango), S $26^{\circ} 44.2^{\prime}, \mathrm{W} 65^{\circ}$ 56.2', 2223 m., 20- 10/2006, at ligth, leg. Gy. Sziráki, E. Horvátha, S. Nuñez Campero.

Pampoconis glencrosi Sziráki, 2009: 170

HOLOTIPO đ̊ (ATNEU028). Argentina, Prov. de Jujuy, Dpto. Ledesma, Calilegua N P, Secondary gallery forest along the Río San Lorenzo, S $23^{\circ} 47.2^{\prime}$, W $64^{\circ} 48.3^{\prime}, 523$ m. a. s. 1., 26/10/2006, leg. Gy. Sziráki, E. Horvát, E. González Olazo. 
Pampoconis xerophila Sziráki, 2009: 172

HOLOTIPO Oิ (ATNEU029). Argentina, Prov. de Catamarca, El Puesta (Caspinchango), semidesert dry scrub, at ligth, S $26^{\circ} 44.2^{\prime}$, W $65^{\circ} 56.7^{\prime}, 2223$ m. a. s. 1., 20- 10/2006, leg. Gy. Sziráki, E. Horvátha, S. Nuñez Campero.

\section{Parasemidalis (Stangesemidalis) enriquei Sziráki, 2009: 183}

PARATIPOS $\hat{o}$ (ATNEU032). Argentina, Catamarca, Los Nacimientos de Abajo, coll. Willink, Terán, Stange. 2 ejemplares.

PARATIPOS $\widehat{o}$ (ATNEU033), 2 ejemplares; PARATIPOS $\hat{\jmath}$ (ATNEU034), 2 ejemplares; PARATIPOS $\widehat{\partial}$ (ATNEU035), 2 ejemplares; PARATIPOS $ð$ (ATNEU036), 2 ejemplares; PARATIPOS $\widehat{\overbrace{}}$ (ATNEU037), 2 ejemplares; PARATIPOS $\hat{\sigma}$ (ATNEU038), 2 ejemplares y PARATIPO + (ATNEU039), 1 ejemplar; Mismos datos que los paratipos (ATNEU032).

PARATIPO $\hat{\text { ô }}$ (ATNEU040). Argentina, La Rioja, Chilecito, 1- 15/I/1969, coll. Willink, Terán, Stange. 1 ejemplar.

PARATIPO ${ }^{\lambda}$ (ATNEU041). Argentina, Salta, Cafayate, Yacochuya, 1950 m. s. n. m., 1- 15/V/1976, coll. Willink, Terán, Stange. 1 ejemplar.

PARATIPOS $\hat{\sigma}$ (ATNEU042), 2 ejemplares y PARATIPOS $\widehat{\partial}$ (ATNEU043), 2 ejemplares Mismos datos que el paratipo (ATNEU041).

Observaciones: en la descripción original, el autor menciona la existencia de 7 paratipos machos de Argentina, Catamarca, Los Nacimientos de Abajo, pero en la colección hay depositados 14 paratipos machos y 1 paratipo hembra. También dice que hay 3 paratipos machos de Argentina, Salta, Cafayate, Yacochuya, pero hay 5 ejemplares.

\section{Semidalis jujuyana Sziráki, 2009:193}

HOLOTIPO $\lesssim$ (ATNEU027). Argentina, Prov. de Jujuy, Dpto. Ledesma, Calilegua N P, Sendero La Lagunita, subtropical deciduous forest, S $23^{\circ} 45.1^{\prime}$, W $64^{\circ} 51.1^{\prime}, 753 \mathrm{~m}$. a. s. 1., 02/11/2006, leg. Gy. Sziráki, E. Horvát, E. González Olazo.

\section{Semidalis maculosus Sziráki, 2009:196}

HOLOTIPO $\widehat{\jmath}$ (ATNEU023). Argentina, Prov. de Jujuy, Dpto. Ledesma, Calilegua N P, Sendero Tataupa, subtropical deciduous forest, $\mathrm{S} 23^{\circ} 44.6^{\prime}, \mathrm{W} 64^{\circ} 51.2^{\prime}, 766 \mathrm{~m}$. a. s. 1., 28/10/2006, leg. Gy. Sziráki, E. Horvát, E. González Olazo.

PARATIPO $\widehat{\jmath}$ (ATENU024). Mismos datos que el holotipo. 1 ejemplar.

PARATIPO $\widehat{\delta}$ (ATENU025). Mismos datos que el holotipo excepto fecha: 27/10/2006. 1 ejemplar.

PARATIPO $\widehat{\jmath}$ (ATENU026). Mismos datos que el holotipo. 1 ejemplar.

\section{Stangesemidalis subandina González Olazo, 1984: 60}

HOLOTIPO ${ }^{\lambda}$ (ATNEU001). R. Argentina, La Rioja, Chilecito, 1- 15/I/1969, Willink, Terán \& Stange coll (Malaise).
ALOTIPO + (ATNEU002). Mismos datos que el Holotipo.

PARATIPOS $\widehat{\partial}$ (ATNEU003), 2 ejemplares y PARATIPO $\widehat{\jmath}$ (ATNEU004), 2 ejemplares, Mismos datos que el Holotipo.

PARATIPO $q$ (ATNEU005). R. Argentina, Catamarca, Los Nacimientos de Abajo, coll. Willink, Terán, Stange. 1 ejemplar.

PARATIPO $\hat{\jmath}$ (ATNEU006), 1 ejemplar; PARATIPO $\hat{\sigma}$ (ATNEU007), 1 ejemplar y PARATIPO $\lesssim$ (ATNEU008), 1 ejemplar, Mismos datos que el Holotipo.

PARATIPO O (ATNEU009). Mismos datos que el paratipo (ATNEU005). 1 ejemplar.

PARATIPO $\widehat{o}$ (ATNEU010), 1 ejemplar y PARATIPO $\widehat{\jmath}$ (ATNEU011), 1 ejemplar, Mismos datos que el Holotipo.

PARATIPO $\widehat{\delta}$ (ATNEU012). R. Argentina, La Rioja, Chilecito, 1- 31/III/1969, Willink \& Stange cols. 1 ejemplar.

PARATIPOS ô y $q$ (ATNEU013). R. Argentina, Salta, Dpto. Anta, Salta Forestal, 50 Km E. J. V. González, 1522/I/1980, col. R. Golbach. 5 ejemplares.

PARATIPO + (ATNEU014). R. Argentina, La Rioja, Chilecito, 13/09/1969, Porter \& Stange cols. 1 ejemplar.

PARATIPOS $q$ (ATNEU015). R. Argentina, Catamarca, 6 Km N. Belén, 1/IV/1969, col. Willink, Terán, Stange. 2 ejemplares.

PARATIPOS $\hat{y} \mathrm{y} q$ (ATNEU016). R. Argentina, Catamarca, 6 Km N. Belén, 1- 15/XII/1968, col. Willink, Terán, Stange (T. Malaise). 17 ejemplares.

PARATIPOS ô y $q$ (ATNEU017). R. Argentina, Salta, Dpto. Anta, Buen Lugar, 40 Km E. Las Lajitas, 02/II/1980, col. R. Golbach. 7 ejemplares.

PARATIPOS $\widehat{\partial} \mathrm{y}$ q (ATNEU018). R. Argentina, Salta, Tacuil, /I/1968, Willink, Terán \& Stange coll. 5 ejemplares.

PARATIPOS o (ATNEU019). Mismos datos que el Holotipo. (Malaise). 7 ejemplares.

PARATIPOS $\delta$ y hembra (ATNEU020). R. Argentina, Catamarca, 6 Km N. Belén, 15- 31/I/1969, Willink, Terán, Stange col. 3 ejemplares.

Observaciones: En la publicación original se detallan 3 paratipos hembras de Argentina, La Rioja, Chilecito, 115/I/1969, pero se encuentran 7. También indica que hay 1 paratipo hembra de Argentina, Salta, Tacuil, pero en la colección hay 3 paratipos machos y 2 paratipos hembras con los mismos datos. Además el autor expresa que en colección en alcohol de la FML están depositados 6 paratipos machos y 5 paratipos hembras de Catamarca, Los Nacimientos de Abajo, pero hay solo 2 paratipos hembra. Además faltan 3 paratipos machos y 1 paratipo hembra de Salta, Yacochuya. Falta 1 paratipo hembra de Argentina, Salta, Dpto. Anta, Buen Lugar, $40 \mathrm{Km}$ E. Las Lajitas. En la publicación original el autor dice haber depositado 2 paratipos machos y 4 paratipos hembras, de Catamarca, $6 \mathrm{Km} \mathrm{N}$. Belén, 1- 15/ XII/1968, pero hay 5 machos y 12 hembras. 


\section{Plecoptera \\ Gripopterygidae}

Neoperla fasciata McLellan et al., 2006: 55

HOLOTIPO $\delta$ (ATPLE004). Argentina, Chubut, La

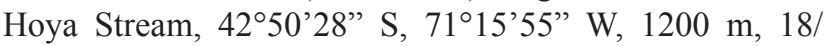
Dec/2004, M. E. Hollmann. Ex Vial P4 (INSUE).

PARATIPOS $\widehat{\partial}$ (ATPLE005), 2 ejemplares y PARATIPOS $\&$ (ATPLE006), 2 ejemplares, Mismos datos que el HOLOTIPO.

PARATIPOS (ATPLE007). Argentina, Chubut, La Hoya Stream, 42॰50'28" S, 71'15'55” W, 1237 m, 23/Nov/2004, M. E. Hollmann. Vial N8 (INSUE). 6 ejemplares.

Neoperla magnaspina McLellan et al., 2006: 59

HOLOTIPO $\widehat{\gamma}$ (ATPLE001). Argentina, Chubut, La

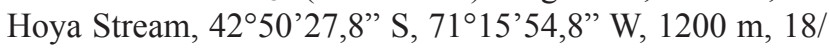
Dec/2004, M. E. Hollmann. Vial P5 (INSUE).

PARATIPO $\bigcirc$ (ATPLE003). Argentina, Chubut, La Hoya Stream, 42 ${ }^{\circ} 50^{\prime} 27,8^{\prime \prime}$ S, 71 ${ }^{\circ} 15^{\prime} 54,8^{\prime \prime}$ W, 1200 m, 18/ Jan/2005, M. E. Hollmann. Vial P7 (INSUE). 1 ejemplar.

\section{Trichoptera \\ Hydrobiosidae}

Iguazu ulmeri Ross and King, 1951: 505

HOLOTIPO ô (ATRI010). R. Argentina, Misiones, Iguazú, 30/I - 13/III/1945, Hayward, Willink y Golbach cols.

\section{Hydroptilidae}

Ithytrichia ferni Rueda Martín, 2006: 252

HOLOTIPO $\widehat{\jmath}$ (ATTRI001). Argentina, Tucumán Prov., Tafí Viejo, Río Tafí, S2643’25”, W64¹7’26”, 827 m., 13/ IV/2005, Rueda Martín col.

PARATIPO (ATTRI002). Argentina, Salta, Santa Victoria, Lipeo, Río Los Naranjos, S22²5'47”, W6444'20", 1109 m., 13/XI/2004, Rueda Martín col. 1 ejemplar (exuvia).

PARATIPO (ATTRI003). Mismos datos que el Holotipo. 4 ejemplares (pupas).

PARATIPO $\delta$ (ATTRI004). Argentina, Tucumán Prov., A ${ }^{\circ}$ El Ceibalito, S2617'6”, W65 31'28', 1210 m., 24/X/1999. 1 ejemplar.

PARATIPO + (ATTRI005). Mismos datos que el Holotipo. 1 ejemplar (pupa).

PARATIPO (ATTRI006). Argentina, Tucumán Prov., Burruyacú, Río del Nío, S26²6’28”, W6459’20”, 1020 m., 07/VII/1999, C. Molineri col. 1 ejemplar (larva).

PARATIPO $q$ (ATTRI007). Mismos datos que el Holotipo. 1 ejemplar (pupa).

PARATIPO (ATTRI008). Mismos datos que el Paratipo (ATTRI002). 6 ejemplares (pupa).

PARATIPO (ATTRI009). Argentina, Tucumán Prov., Tafí Viejo, Río Tafí, 10/X/2006, Rueda Martín col. 14 ejemplares (pupa).

Observaciones: en la publicación original se detallan 7 pupas y 8 larvas de Argentina, Salta, Santa Victoria, pero en la colección hay 6 pupas y 1 exuvia. Además, se aclara que hay 2 pupas y 1 metamorfotipo macho de Argentina, Tucumán Prov., Tafí Viejo, Río Tafí, pero en cambio hay 4 pupas. También hay 14 pupas de Argentina, Tucumán Prov., Tafí Viejo, Río Tafí, con una fecha distinta que no aparecen en la publicación original.

\section{Zygentoma \\ Lepismatidae}

Sceletolepisma pauliani (Wygodzinsky, 1959): 442

PARATIPO $\widehat{\diamond}$ (ATZIG016). South Africa, Namib, Sandwich Bay, 13/V/1959, R. Paulian (col.). 1 ejemplar.

Stachisma mexicana Wygodzinsky, 1949b: 224

PARATIPOS (ATZYG001). México, Baja California, Isla Natividad, 5/6/1945, B. F. Osorio col. 2 ejemplares.

\section{Meinertellidae}

Machilinus birabeni Wygodzinsky, 1944: 91

ALOTIPO $q$ (ATZYG015). R. A., Río Negro, Bariloche, 06/02/1949, Kusnezov col.

Observaciones: en la descripción original, el autor solo describe al macho de esta especie y define al Holotipo de Chubut, Gorbenador Costa. En la colección se encuentra el Alotipo hembra.

Machilinus inopinatus Wygodzinsky, 1951: 437

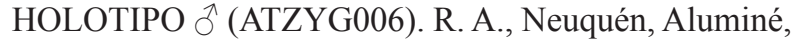
19/01/1949, María y F. Monrós col.

ALOTIPO (ATZYG007). Mismos datos que el Holotipo.

PARATIPOS $\delta^{\wedge} \mathrm{y}$ ㅇ (ATZYG008). Mismos datos que el Holotipo. 6 ejemplares.

Machilinus muntañolae Wygodzinsky, 1949c: 598

HOLOTIPO $\lesssim$ y ALOTIPO + (ATZYG002). R. A., Neuquén, Hua Hum, 27/I/1949, Monrós col.

PARATIPOS $q$ (ATZYG003). Chubut, Lago Verde, 16/02/1949, Monrós col. 2 ejemplares.

PARATIPOS (ATZYG004). R. A., Neuquén, Hua Hum, 22/I/1949, Monrós col. 6 ejemplares.

PARATIPOS $q$ (ATZYG005) R. A., Neuquén, Moquehué, 22/01/1949, Monrós col. 2 ejemplares.

\section{Nicoletiidae}

Anelpistina ramosi (Wygodzinsky, 1959): 447

HOLOTIPO $\widehat{\jmath}$ y ALOTIPO + (ATZYG017). Cuba, Cueva Grande, Punta Término de Yaguajay de las Villas, Siñva Taboada col.

Anelpistina weyrauchi (Wygodzinsky, 1959): 450

HOLOTIPO đ̊ y ALOTIPO + (ATZYG018). Perú, Lima, Atocongo, 250 mts., 11/09/1949, W. K. W. col., Leg. Weyrauch.

Attatelura aethyctera Wygodzinsky, 1958: 116

HOLOTIPO $\widehat{~}$ (ATZYG009). R. A., Tucumán, con So- 
lenopsis, 25/01/1954, Golbach leg.

ALOTIPO $q$ (ATZYG010). Mismos datos que el Holotipo.

PARATIPO (ATZYG011). Mismos datos que el Holotipo. 1 ejemplar.

Observaciones: el autor, en la descripción original enumera 3 paratipos machos, 2 paratipos hembras de la misma localidad y fecha que el holotipo, además dice que hay 1 paratipo macho de Tucumán, 15/XII/1949, pero en la colección solo hay depositado 1 paratipo macho.

Grassiella carioca Wygodzinsky, 1958: 105

HOLOTIPO đ̄ (ATZYG012). Brasil, Río de Janeiro, D. F., 21/07/1941, J. Marianek col.

ALOTIPO $q$ (ATZYG013). Mismos datos que el Holotipo.

PARATIPO đ̋ (ATZYG014). Brasil, Río de Janeiro, Leme, D.F., 20/VI/1941. 1 ejemplar.

\section{AGRADECIMIENTOS}

Deseo agradecer a Sonia Piroski (Biblioteca de la Sociedad Entomológica Argentina), Carlos Carabajal y María Luisa Maciel Talavera (Centro de Información Geo- Biológico del NOA, FML) por facilitarme la bibliografía solicitada. Al Sr Editor José Luis Navarrete-Heredia y los Sres. Árbitros por sus oportunas sugerencias.

\section{LITERATURA CITADA}

Bustillo Pardey, A. E. y Z. N. Gil Palacios. 2008. Capítulo

2: Características de la clase Insecta. (pp. 21 - 33). En: Bustillo Pardey, A.E. (Ed.). Los insectos y su manejo en la caficultura colombiana, Cinchiná, Colombia.

Córdoba, S.P. 2019. Catálogo de los especímenes tipo de Ephemeroptera (Insecta) depositados en la Colección Entomológica Conservada en Alcohol del InstitutoFundación Miguel Lillo, Tucumán, Argentina. Revista de la Sociedad Entomológica Argentina, 78(4): 37-54. https://doi.org/10.25085/rsea.780406

Fidalgo, P. 1989. Revisión de las especies neotropicales del género Acmoplynema Ogl. (Hymenoptera: Mymaridae). Revista de la Sociedad Entomológica Argentina, 46(14): 3-67.

González Olazo, E.V. 1984. Stangesemidalis subandina, nuevo género y especie de Coniopterygidae de la República Argentina (Neuroptera, Planipennia). Acta Zoológica Lilloana, 38(1): 59-63.

Guzmán- Mendoza, R., J. Calzontzi- Marín, M.D. SalasAraiza y R. Martínez- Yañez. 2016. La riqueza biológica de los insectos: análisis de su importancia multidimensional. Acta Zoológica Mexicana, 32(3): 370- 379.

Hayward, K.J. 1963. Los tipos de Insecta (exceptuando Diptera) conservados en el Instituto Miguel Lillo. Acta Zoológica Lilloana, 19: 297-334.
Llorente-Bousquets, J., S.E. González, A.N. García-Aldrete y C. Cordero. 1996. Breve panorama de la taxonomía de artrópodos en México (pp. 3-14). En: Llorente, J., A.N. García y E. González (Eds.). Biodiversidad, taxonomía y biogeografía de artrópodos de México: hacia una síntesis de su conocimiento. CONABIO-UNAM, México.

McLellan, I., M.L. Miserendino and M.E.T. Hollmann. 2006. Two new species of Notoperla (Plecoptera: Gripopterygidae) and a redescription of Notoperlopsis femina Illies. Zootaxa, 1140: 53-68. https://doi. org/10.11646/zootaxa.1140.1.3

Purvis, A. and A. Hector. 2000. Getting the measure of biodiversity. Nature, 105: 212-219. https://doi. org/10.1038/35012221

Roisin, Y. 2003. Cryptotermes chacoensis, a new species from native south american inland habitats (Isoptera: Kalotermitidae). Sociobiology, 42(2): 319-327.

Ross, H.H. and E.W. King. 1951. A key to the world genera of the Caddisfly tribe "Hydrobiosini" (Trichoptera, Rhyacophilidae). Acta Zoológica Lilloana, 12: 501-515.

Rueda Martín, P.A. 2006. New record of the genus Ithytrichia (Trichoptera: Hydroptilidae) for South America, with descriptions of male, larva and pupa of a new species from northwestern Argentina. Aquatic Insects, 28(4): 251-256. https://doi.org/10.1080/01650420601072276

SZiráki, G. 2009. Data on Coniopterygidae of Argentina, with subgeneric division of Parasemidalis Enderlein, 1905. Folio Historico Naturalia Musei Matraensis, 33: 169-199.

Wygodzinsky, P. 1944. Contribuicao ao conhecimento da familia Machilidae (Thysanura) do Mexico, do Brasil e da Argentina. Revista de Entomología, 15: 54-96.

Wygodzinsky, P. 1949a. Contribuciones al conocimiento de los Simuliidae argentinos. II. Gigantodax horcotiani sp. n., de la Provincia de Tucumán (Diptera). Anales de Instituto Medico Regional, 2(3): 325-333.

Wygodzinsky, P. 1949b. Contribución al conocimiento de las Lepismatinae americanas (Lepismatidae, Thysanura). Acta Zoológica Lilloana, 6: 215-227.

Wygodzinsky, P. 1949c. Notas y descripciones de algunas Machilidae americanas (Thysanura, Insecta). Acta Zoológica Lilloana, 7: 593-622.

Wygodzinsky, P. 1951. Apuntes sobre Thysanura americanas (Apterygota, Insecta). Acta Zoológica Lilloana, 11: 435-458.

Wygodzinsky, P. 1958. Sobre algunos «Nicoletiidae» americanos (Thysanura, Insecta). Acta Zoológica Lilloana, 16: 97-120.

Wygodzinsky, P. 1959. Contribution to the knowledge of the "Thysanura" and "Machilidae" (Insecta). Revista Brasileira de Biología, 19: 441-457. 
Zumbado, M.A. y Azofeifa, D. 2018. Parte I, Introducción a los insectos. (pp. 13-37). En: Programa Nacional de Agricultura Orgánica (PNAO). Insectos de importancia agrícola. Guía básica de Entomología. Heredia, Costa Rica.

Recibido: 12 marzo 2021

Aceptado: 1 mayo 2021
Zwick, P. 2007. Some net-winged midges from Argentina (Diptera: Blephariceridae). Studies on Neotropical Fauna and Environment, 42(2): 109-119. https://doi. org/10.1080/01650520600908436 